\title{
Modeling of grain processing in a pneumomechanical dresser
}

\author{
Elmas Nurullin*, Ravil Ibyatov, Andrey Dmitriev, and Damir Khaliullin \\ Kazan State Agrarian University, 420015 Kazan, Russia
}

\begin{abstract}
To improve the quality and effectiveness of pre-sowing treatment of seeds of grain crops, a hypothesis was put forward on the possibility of using the air flow to continuously perform a set of technological operations in the process of dressing. The proposed approach is implemented in a unrivalled pneumomechanical deresser, in the processing chamber of which a multiphase flow of dispersed mixture "air-seeds-droplet" is formed. For the theoretical justification of the main parameters of the processing process, a mathematical model of the movement of seeds and fine droplets of the working fluid was developed and numerically solved taking into account the decrease in their concentration along the length of the working chamber. As a result, it was found that the speeds of seeds and drops of different sizes as they move in the processing chamber naturally increase, and the concentration of drops decreases asymptotically. It was revealed that to ensure high quality processing at the entrance to the processing chamber, the seeds should move in suspension with the speed of the corresponding droplet speed of the working fluid. The minimum path length of the "air-seed-droplet" mixture is substantiated, where the maximum decrease in the concentration of drops occurs due to the adhesion of seeds suspended in the air flow to the surface. The results obtained are important for optimizing the length of the processing chamber, substantiating the modes and parameters of the movement of air, grain and drops, at which full coverage of seeds with a minimum flow rate of the working fluid is achieved. In the long term, the research results will serve as the basis for optimizing the design and technological parameters of pneumomechanical dressers, which ensure high quality and efficiency of pre-sowing treatment of seeds of grain crops.
\end{abstract}

\section{Introduction}

In modern grain production technologies, pre-sowing seed treatment (dressing) with liquid working solutions of chemical and biological protective and stimulating agents occupies an important place in order to protect them from diseases, pests and stimulate vigorous initial development in the soil. The quality and effectiveness of pre-sowing treatment mainly depends on the dressing method and the design of the presowing treatment machine [1-3].

The analysis of scientific and technical literature and patent studies show that in modern seed dressers presowing treatment is carried out in a closed chamber by applying a working solution in a small drop form on seeds scattered throughout the chamber volume [4-12].

It is also known that pneumatic systems that are used to perform various technological operations have found widespread use in agricultural machines [13-17].

Based on these studies, a hypothesis was put forward on the possibility of using the air flow in seed dressers to perform a complex of technological operations in a single continuous stream, including the creation of a suspended state of seeds in the processing zone, which provides coverage of each grain with a working fluid.

The proposed approach is implemented in pneumomechanical dressers that have no analogues in the world $[18,19]$. One of the main structural elements of the pneumomechanical seed dresser is the treatment chamber, where fine drops of the working fluid are applied to seeds moving in the air stream.

To justify the main design parameters of the processing chamber of the pneumomechanical treater, providing the highest performance indicators, it is necessary to simulate the processing process taking into account all the main factors affecting the process.

The purpose of the study is to simulate the process of pre-sowing treatment of seeds of grain crops in a pneumomechanical dresser.

\section{Materials and methods}

The processing chamber of the pneumomechanical dresser is a horizontal pipeline of a certain diameter. At the entrance of the air-grain mixture into the processing chamber, the working fluid in the form of fine particles (drops) is injected into it. A multiphase dispersed mixture "air-seeds-drop" is formed in the chamber, during the movement of which the seeds are enveloped with a working fluid. The more seeds are completely and evenly enveloped, the higher will be the quality of processing, the effectiveness of its action at the lowest flow rate of the working fluid. These indicators are affected by masses, velocities, geometric parameters,

* Corresponding author: nureg@mail.ru 
concentration of seeds and fine particles of the protective fluid, air velocity and density, time of movement of the multiphase air-seed-drop mixture.

The condition for the movement of a multiphase dispersed mixture in a processing chamber allows implementing the methodological foundations of the dynamics of multiphase media for mathematical modeling of seed surface coating with a working fluid [20]. In the research, works in the field of modeling the movement of air-grain and other multiphase mixtures in various working channels and pneumatic systems of agricultural machines were also used [21-23].

The methodological approaches described in these works suggest that when the size of the dispersed composition of a multiphase mixture is much larger than the molecular level and the particles themselves are much smaller than the distances at which the averaged and macroscopic parameters of the mixture change significantly, mathematical models of the motion of dispersed particles of the mixture can be built on differential equations. The following assumptions were made: there is no heat transfer between the dispersed particles; temperature and air density are constant; viscosity of air is not taken into account, particles have a spherical shape.

Based on the above materials, we constructed a mathematical model of the coating process for seeds moving in suspension in the air flow with finely divided drops of the working fluid based on the differential equations of their movement in the processing chamber. At the same time, we took into account that due to the deposition of fine droplets on the surface of the seeds, their concentration decreases along the length of the chamber.

Let us consider the process of joint movement of seeds and fine droplets of the working fluid in the air flow (Fig. 1).

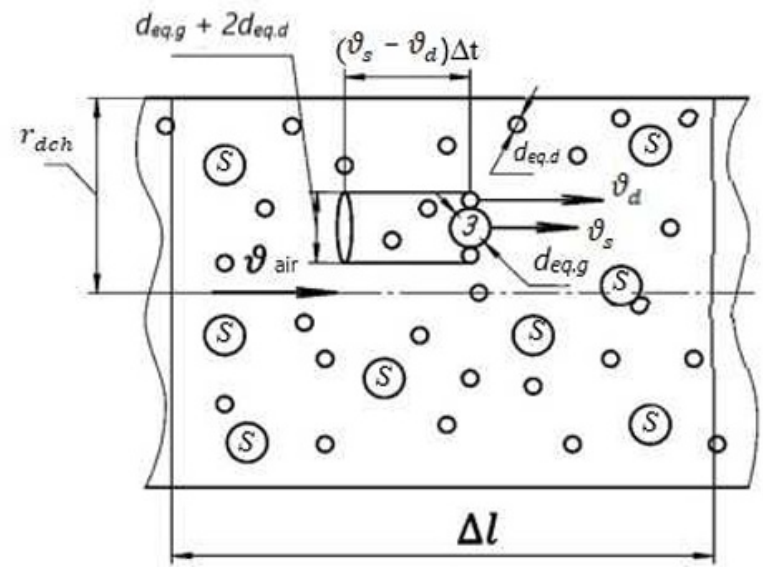

Fig. 1. Dressing process modeling: o are the particles of the working fluid; (S) are seeds

The axial air velocity in the initial section is denoted as $\vartheta_{\text {air }}$. The axial speeds of seeds and fine droplets of the working fluid in the feed zone into the treatment chamber are zero. Under the action of moving air, they begin to gain speed and accelerate to their asymptotic values, depending on the windage coefficients.
The movement of seeds and fine droplets in the air stream can be described using the following differential equations:

$$
\begin{aligned}
& m_{s} \frac{d \vartheta_{s}}{d t}=\frac{k_{s} S_{s} \rho_{\text {air }}}{2}\left|\vartheta_{\text {air }}-\vartheta_{g}\right| \cdot\left(\vartheta_{\text {air }}-\vartheta_{g}\right), \\
& m_{d} \frac{d \vartheta_{d}}{d t}=\frac{k_{d} S_{d} \rho_{\text {air }}}{2}\left|\vartheta_{\text {air }}-\vartheta_{d}\right| \cdot\left(\vartheta_{\text {air }}-\vartheta_{d}\right),
\end{aligned}
$$

where $m_{g}, m_{d}$ are respectively, the mass of seeds and fine drops of the working fluid, $\mathrm{kg}, \vartheta_{s}, \vartheta_{d}, \vartheta_{\text {air }}$ respectively, the speed of seeds, fine drops of the working fluid and air, $\mathrm{m} / \mathrm{s} ; t$ is the time of movement, $\mathrm{s}$; $k_{g}, k_{d}$ is the coefficient of resistance of seeds and fine droplets of the working fluid; $S_{g}, S_{d}$ are the mid-sections of grain and fine droplets of the working fluid, $\mathrm{m}^{2} ; \rho_{\text {air }}$ is air density, $\mathrm{kg} / \mathrm{m}^{3}$.

The masses and areas of mid-sections of seeds and drops are determined by the following formulas through their equivalent diameters:

$$
\begin{gathered}
S_{g}=\frac{\pi d_{\text {eq.g }}^{2}}{4}, \quad S_{\mathrm{K}}=\frac{\pi d_{\text {eq.d }}^{2}}{4}, \\
m_{g}=\frac{\pi d_{\text {eq.g }}^{3}}{6} \rho_{g}, \quad m_{d}=\frac{\pi d_{\text {eq.d }}^{3}}{6} \rho_{d},
\end{gathered}
$$

where $d_{\text {eq.g }}, d_{\text {eq.d }}$ are, respectively, the equivalent diameters of seeds and drops of the working fluid, m; $\rho_{g}$, $\rho_{d}$ are, respectively, the density of seeds and fine drops of the working fluid, $\mathrm{kg} / \mathrm{m}^{3}$.

After the transition to the equivalent diameters of grain and fine droplets, taking into account the fact that the air flow rate is greater than the velocities of grain and droplets, equations (1) and (2) will take the following form:

$$
\begin{aligned}
\frac{d \vartheta_{g}}{d t} & =\frac{4}{3} k_{g} \frac{\rho_{\text {air }}}{\rho_{g} d_{\text {eq.g }}}\left(\vartheta_{\text {air }}-\vartheta_{g}\right)^{2}, \\
\frac{d \vartheta_{d}}{d t} & =\frac{4}{3} k_{d} \frac{\rho_{\text {air }}}{\rho_{d} d_{\text {eq.d }}}\left(\vartheta_{\text {air }}-\vartheta_{d}\right)^{2} .
\end{aligned}
$$

When solving equations (5) and (6), the air flow rate is considered known. However, it is not constant. To determine the change in air-grain flow velocity, we write the integral equation for maintaining the material balance of the medium:

$$
\begin{gathered}
\int_{0}^{r_{d c h}} 2 \pi r_{d c h} \mu_{\text {air }} \rho_{\text {air }} \vartheta_{\text {air }} d r_{d c h}+ \\
+\int_{0}^{r_{d c h}} 2 \pi r_{d c h} \mu_{g} \rho_{g} \vartheta_{g} d r_{d c h}+ \\
+\int_{0}^{r_{d c h}} 2 \pi r_{d c h} \mu_{d} \rho_{d} \vartheta_{g} d r_{d c h}=Q,
\end{gathered}
$$

where $Q$ is the sum of the mass flow rates of air, seeds and fine droplets of the working fluid; $r_{d c h}$ is the radius of the preliminary dressing chamber, $\mu_{\text {air }}, \mu_{g}, \mu_{d}$ are the volumetric concentrations of air, seeds and fine droplets of the working fluid, $\mathrm{m}^{3} / \mathrm{m}^{3}$.

After calculation, the integral equation (7) will take the following form:

$$
\begin{gathered}
\pi r_{d c h}{ }^{2} \mu_{\text {air }} \rho_{\text {air }} \vartheta_{\text {air }}+\pi r_{d c h}{ }^{2} \mu_{g} \rho_{g} \vartheta_{g}+ \\
+\pi r_{d c h}{ }^{2} \mu_{d} \rho_{d} \vartheta_{d}=Q
\end{gathered}
$$

From here we get the following formula for determining the air velocity:

$\vartheta_{\text {air }}=\frac{Q}{\pi r_{d c h}{ }^{2} \mu_{\text {air }} \rho_{\text {air }}}-\frac{\mu_{g} \rho_{g}}{\mu_{\text {air }} \rho_{\text {air }}} \vartheta_{g}-\frac{\mu_{d} \rho_{d}}{\mu_{\text {air }} \rho_{\text {air }}} \vartheta_{d}$. 
The flow in question is a multiphase mixture with two dispersed phases. When they collide, fine particles of working liquid are deposited on the surface of the seeds. Therefore, the concentration of fine droplets $\mu_{d}$ is a variable that decreases in time.

The value of the reduced concentration of atomized particles, time dt, can be represented as

$$
\sigma(t)=\mu_{d}(t)-\mu_{d}(t+\Delta t) .
$$

The volume and weight of the deposited droplets are taken into account in the characteristics of the solid phase and air flow in the following ratio:

$$
\rho_{g}(t+\Delta t)=\mu_{g} \rho_{g}-\sigma(t) \rho_{d}
$$

For further theoretical studies, it is necessary to determine the total volumes of seeds and drops in the treatment chamber.

The total volume of fine droplets of the working fluid at the working section of the treatment chamber with a length $\Delta l$ (Fig. 1) is:

$$
V_{v d}(t)=\pi r_{d c h}^{2} \Delta l \mu_{d}(t)
$$

We calculate the number of drops in the considered volume of the treatment chamber:

$$
N_{v d}(t)=\frac{V_{v d}(t)}{V_{d}} .
$$

Similar formulas can be written for the total volume and number of seeds in the considered volume of the treatment chamber:

$$
\begin{gathered}
V_{v g}(t)=\pi r_{d c h}{ }^{2} \Delta l \mu_{g}(t) . \\
N_{v g}(t)=\frac{V_{v g}(t)}{V_{g}} .
\end{gathered}
$$

Let us consider the frontal blowing of one grain with a stream of air and fine droplets of a protective liquid (Figure 1). Over time $\Delta t$, all drops hit the grain kernels in the elementary cylinder with a diameter $d_{e q . g}+2 d_{\text {eq.d }}$ and a length $\left(\vartheta_{g}-\vartheta_{d}\right) \Delta t$, the volume of which is equal to:

$$
V_{c}=\frac{\pi}{4}\left(d_{\text {eq.g }}+2 d_{\text {eq.d }}\right)^{2} \cdot\left(\vartheta_{g}-\vartheta_{d}\right) \Delta t .
$$

Let us calculate the number of drops in the elementary cylinder deposited on one grain:

$$
n(t)=\frac{3\left(d_{e q . g}+2 d_{e q . d}\right)^{2} \cdot\left(\vartheta_{g}-\vartheta_{d}\right) \Delta t \mu_{d}}{2 d_{e q . d}^{3}} .
$$

The volume of the working fluid deposited in the form of droplets on the surface of the grain over time on the section $\Delta l$ of the working zone of the dressing chamber is determined as follows:

$$
V_{\mathrm{p} d}(t)=\frac{\pi\left(d_{e q . g}+2 d_{e q . d}\right)^{2}}{4} \cdot\left(\vartheta_{g}-\vartheta_{d}\right) \Delta t \mu_{d} N_{v g}(t) .
$$

We write down the condition for preserving the number of drops of the protective liquid in the section of the etching chamber with a length $\Delta l$ :

$$
V_{v d}(t+\Delta t)-V_{v d}(t)=V_{\mathrm{p} d}(t) \text {. }
$$

Hence, taking into account equations (11), (14), (17), after substitution and reductions, we obtain:

$$
\begin{gathered}
\mu_{d}(t+\Delta t)-\mu_{d}(t)=\frac{3\left(d_{\text {eq.g }}+2 d_{\text {eq.d }}\right)^{2}}{2} x \\
x\left(\vartheta_{g}-\vartheta_{d}\right) \Delta t \mu_{d} \frac{\mu_{g}}{d_{\text {eq.g }}^{3}} .
\end{gathered}
$$

Hence, using the formula for the derivative $\mu_{d}^{\prime}=\lim _{\Delta t \rightarrow 0} \frac{\mu_{\mathrm{d}}(\mathrm{t}+\Delta \mathrm{t})-\mu_{\mathrm{d}}(\mathrm{t})}{\Delta \mathrm{t}}$, we obtain the following differential equation for calculating the change in the average concentration of the working fluid droplets over time:

$$
\frac{d \mu_{d}}{d t}=\frac{3\left(d_{e q . g}+2 d_{e q . d}\right)^{2}}{2 d_{e q . g}^{3}}\left|\vartheta_{g}-\vartheta_{d}\right| \mu_{d} \mu_{g} .
$$

Over time $\Delta t$, the drop moves along the distance $\Delta l=$ $\vartheta_{d} \Delta t$, i.e. $d t=d l / \vartheta_{d}$. Then in equation (20) we can shift to coordinates $l$ :

$$
\frac{d \mu_{d}}{d l}=\frac{3\left(d_{e q . g}+2 d_{e q . d}\right)^{2}}{2 d_{e q . g}^{3} \vartheta_{d}}\left(\vartheta_{g}-\vartheta_{d}\right) \mu_{d} \mu_{g} .
$$

Thus, as a result of mathematical modeling, differential equations (5), (6), (21) were obtained that describe the process of coating seeds of grain crops with fine particles of the working fluid in the processing chamber, taking into account a decrease in their concentration. Differential equations (5), (6), (21) were solved in the MATLAB software package using the numerical method under the following initial conditions: $t=0 ; \quad \vartheta_{g}=0 ; \quad \vartheta_{d}=0 ; \quad \mu_{d}=\mu_{d}^{i}$, where $\mu_{d}^{i}$ is the concentration of fine droplets of the working fluid at the time of injection into the treatment chamber. Due to the fact that the air flow rate is not constant, at each step of numerical integration, it is refined by equation (9).

\section{Results and discussion}

The numerical calculations of changes in seed velocities of various sizes were performed according to differential equation (5) with an equivalent diameter of atomized particles of a working fluid of $25 \mu \mathrm{m}\left(\mathrm{d}_{\text {eq.d }}=25 \mu \mathrm{m}\right)$. This value of equivalent diameter corresponds to the average particle size during aerosol treatment, at which the maximum efficiency of coating the surface of the seeds and the penetration of the working solution into micropores and microdamages is achieved.

The results of calculations of changes in seed velocity in graphical form are presented in Fig. 2.

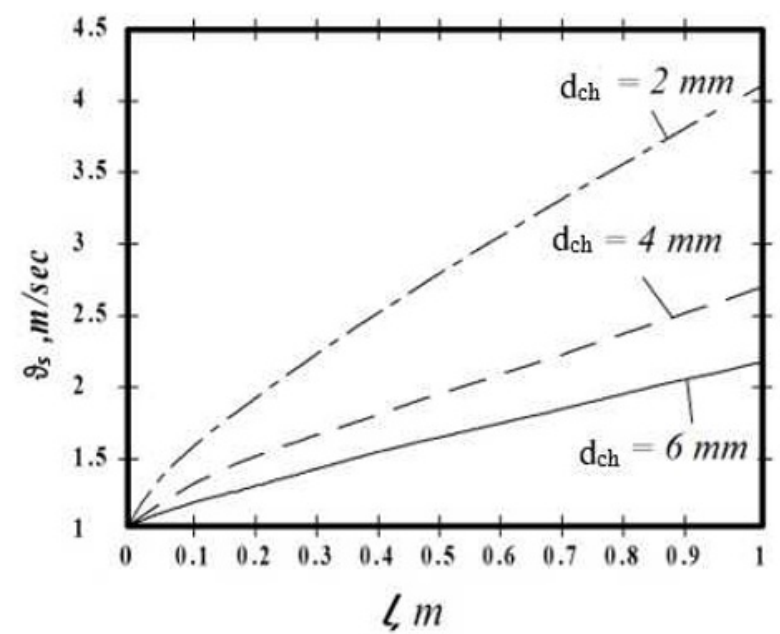

Fig. 2. Seed velocity change in the preliminary chamber (for an equivalent diameter of sprayed particles $d_{\text {eq.d. }}=25 \mu \mathrm{m}$ ).

The calculations of the change in the velocities of the sprayed particles of the working fluid of various sizes were performed according to differential equation (6) with an equivalent seed diameter of $4 \mathrm{~mm}\left(\mathrm{~d}_{\text {eq. }}=4 \mathrm{~mm}\right)$. 
This value of equivalent diameter corresponds to the average seed size of the main crops. The results of calculations of changes in droplet velocities are presented in graphical form in Fig. 3.

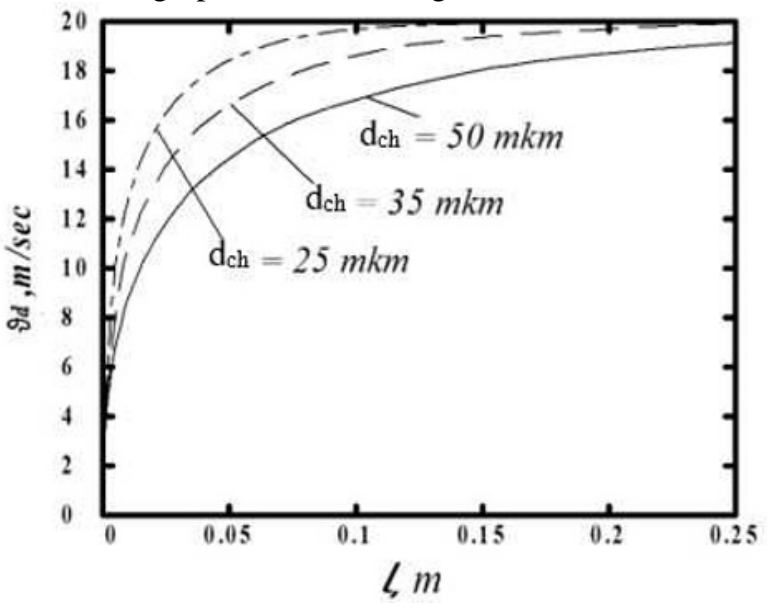

Fig. 3 Velocity alteration of the working fluid particles in the preliminary chamber (for the equivalent diameter of seed $\mathrm{d}_{\text {eq. }}=4 \mathrm{~mm}$ )

Let us analyse the results presented in Figures 2 and 3.

Evidently from Fig. 2, the velocities of seeds increase constantly in the process of movement in the working chamber. Such dynamics is inherent in all values of seed sizes at the accepted value of the equivalent diameter of a working fluid drop $(\mathrm{d}=25 \mu \mathrm{m})$. Moreover, the speed of small seeds increases faster. Obviously, the same pattern will be observed for all sizes of droplets of the working fluid.

Analysis of the graphs presented in Fig. 3 shows that droplet velocities of various diameters increase very rapidly. Drops accelerate to their asymptotic values $18-20 \mathrm{~m} / \mathrm{s}$ ) along a path of $0.15-0.25$ meters. This pattern is observed for all droplet sizes with the accepted value of the equivalent grain diameter. Obviously, it will be observed for seeds of all sizes.

A comparison of the graphs presented in Figures 2 and 3 shows that when achieving maximum values of the drop velocities $(18-20 \mathrm{~m} / \mathrm{s})$, the velocities of the seeds is just $1.5-2.0 \mathrm{~m} / \mathrm{s}$. At this speed, the seeds move unsuspended in the treatment chamber, which does not provide complete enveloping of each grain with fine particles of the working fluid. Therefore, the seeds at the entrance to the processing chamber must have a speed that ensures their movement in suspension (15-20 m/s), which is comparable to the speed of fine particles of the working fluid. This creates the conditions for the effective interaction of fine droplets with the surface of each seed, which provides a high degree of seed coverage with the agent at a minimum length of the dressing chamber. It should also be noted that the seeds in the process of moving in the air stream in the environment of fine particles of the working fluid take different positions, which further contributes to the uniform coating of the entire surface of the seeds with the working fluid.

When substantiating the length of the processing chamber, it is necessary to take into account the decrease in the concentration of fine droplets of the working fluid in it as the multiphase dispersed mixture "air-seedsdrop" moves. It is important to know at what length of displacement the complete flow of the working fluid drops occurs from the moment of supplying the finely divided drops of the working fluid into the flow of the moving air-grain mixture. As the air-grain-drop mixture moves in the dressing chamber, the concentration of droplets will decrease due to settling on the surface of the seeds. Numerical calculations of the change in the concentration of the working fluid in the treatment chamber were carried out according to equation (21) with an equivalent diameter of the droplets of the working fluid of $25 \mu \mathrm{m}\left(\mathrm{d}_{\text {eq. }}=25 \mu \mathrm{m}\right)$ and various seed sizes. The results of these calculations in the form of graphs are shown in Fig. 4.

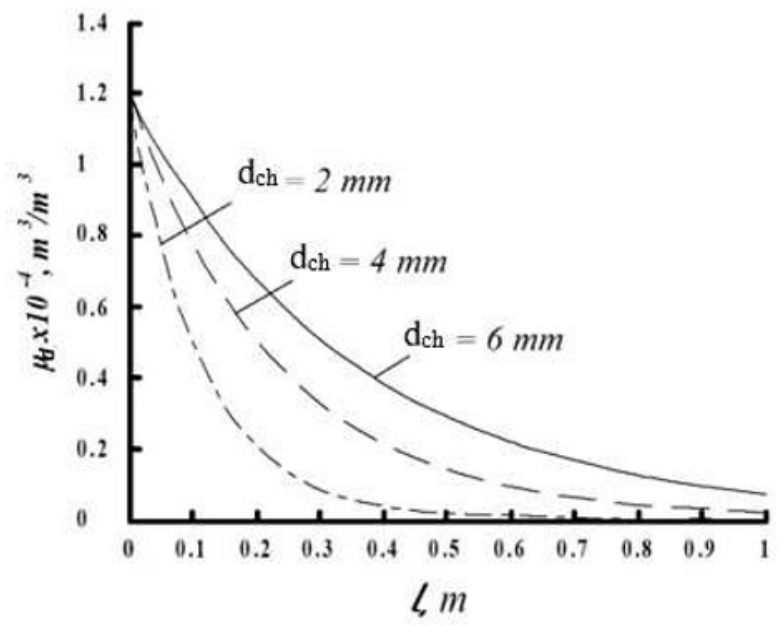

Fig. 4. Change of the concentration of sprayed particles of the working fluid in the preliminary chamber depending on the equivalent diameter of seed (for the equivalent diameter of sprayed particles $\mathrm{d}_{\mathrm{eq}}=25 \mu \mathrm{m}$ ).

Analysis of the plots in Fig. 4 shows that the concentration of droplets of the working fluid along the length of the treatment chamber decreases asymptotically. This pattern is observed for all seeds with the difference that for small seeds the concentration decreases more intensively than for large seeds. This suggests that the total coating surface of small seeds is larger, so more particles of the working fluid settle on their surface. Obviously, the resulting pattern will be observed with other values of the size of the droplets of the working fluid. When treating small seeds $\left(\mathrm{d}_{\mathrm{g}}=2 \mathrm{~mm}\right)$, the concentration of the droplets of the working fluid drops to zero when the multiphase dispersed mixture "air-seeds-drop" moves to a distance of $0.8-0.9 \mathrm{~m}$. For medium-sized seeds $\left(\mathrm{d}_{\mathrm{g}}=4 \mathrm{~mm}\right)$ the concentration of droplets of the working fluid within the specified boundary conditions of numerical calculations (the length of the multiphase mixture is one meter) decreases by $97-98 \%$ and by $92-94 \%$ for large grains $\left(\mathrm{d}_{\mathrm{g}}=6 \mathrm{~mm}\right)$.

Summarizing the results of modeling the process of seed treatment in the working chamber of a pneumomechanical seed dresser and their numerical solution, it should be noted that they are consistent with the hypothesis of the study. 
The results obtained are of practical importance in substantiating the length of the treatment chamber at which the maximum decrease in the concentration of droplets of the working fluid occurs due to their deposition on the seeds (adhesion to the surface of the seeds), as well as other structural and technological parameters of the pneumomechanical treating agent. Settling the maximum number of drops on the surface of the seeds means using the entire dose of the working fluid and coating the surface of each seed, which ensures high quality seed treatment and eliminates the cost overrun of expensive protective-stimulating agents.

The numerical solution of the differential equation describing the process of decreasing the concentration of drops of the working fluid indicates that not all drops are deposited on the surface of the seeds on the length of the working chamber of $1.0 \mathrm{~m}$ when processing medium and large grains. However, obviously from Fig. 4, for medium and large seeds, the graphs asymptotically approach the abscissa axis. Therefore, by increasing the length of the chamber to a certain value, it is possible to achieve the sedimentation of all drops on the surface of the seeds.

On the other hand, such a small number of unspent drops of the working fluid (2-3\% of medium- seeds and 6-8 \% of large seeds) will settle on the seeds when moving the mixture to the unloading hopper designed to collect the treated seeds and separate them from the air flow. In addition, the length of the processing chamber can be limited by overall dimensions and other design requirements of the pneumomechanical treater.

In order to fully confirm the hypothesis put forward that it is possible to use the air flow in seed dressers to perform several seed loading operations in a single continuous process stream while simultaneously eliminating dust and creating the stream suspending seeds in the air, which ensures that each grain is coated with a working fluid, in the future it is necessary carry out studies to substantiate the speed of the air flow, providing a balanced state of seeds at the entrance to the processing chamber.

\section{Conclusion}

Based on an analysis of research in the field of presowing treatment of seeds of grain crops, a hypothesis was put forward that it is possible to use the air flow in the seed dressers to carry out technological operations for loading and feeding seeds into the treatment chamber in a single continuous stream, creating seeds suspended in the air flow, which ensures that each grain is coated with a working fluid.

As a result of the studies, differential equations were obtained that describe the process of coating seeds of grain crops with finely dispersed drops of protective liquid during the movement of a multiphase air-graindrop mixture in the treatment chamber of a pneumomechanical dresser, the numerical solution of which made it possible to reveal patterns of change in the speeds of grain and drops of various sizes, also a decrease in the concentration of drops as the mixture moves in the working area.

It was found that the velocity of seeds and drops as they move in the processing chamber naturally increase for all sizes. It was found that the droplets accelerate to asymptotic values of velocities $(18-20 \mathrm{~m} / \mathrm{s})$ along the path length of $0.15-0.25$ meters, and the velocity of the seeds at the same distance is only $1.5-2.0 \mathrm{~m} / \mathrm{s}$. These velocities do not provide a balanced state of seeds in the air flow, respectively, the complete enveloping of each grain with a protective fluid. Therefore, the seeds at the entrance to the processing chamber must have a velocity that ensures their movement in suspension $(15-20 \mathrm{~m} / \mathrm{s})$, which creates the conditions for the effective interaction of fine droplets with the surface of each seed.

It was determined that the most intense decrease in droplet concentration occurs along a path length of 0.8-0.9 meters: when treating small seeds with an equivalent diameter of $2 \mathrm{~mm}\left(\mathrm{~d}_{\mathrm{g}}=2 \mathrm{~mm}\right)(100 \%)$; medium seeds $\left(d_{g}=4 \mathrm{~mm}\right)(97-98 \%)$ and large seeds $\left(\mathrm{d}_{\mathrm{g}}=6 \mathrm{~mm}\right)(92-94 \%)$. In this case, concentration reduction plots for medium and large seeds (Fig. 4) asymptotically approach the abscissa axis. This gives reason to assert that with an increase in the path (length of the working chamber) all drops will adhere to the surfaces of the seeds.

The research results serve as the basis for recommendations and further studies on optimizing the constructive and technological parameters of seed dressers based on the use of an air stream to perform a set of operations in a single continuous stream, ensuring the quality and high efficiency of pre-sowing treatment of grain seeds.

\section{References}

1. R.R. Khamaletdinov, I.I. Gabitov, S.G. Mudarisov, E.R. Khasanov, V.M. Martynov, A.V. Negovora, V.A. Stupin, F.N. Gallyamov, I.M. Farkhutdinov, D.Y. Shirokov, Improvement in Engineering Design of Machines for Biological Crop Treatment with Microbial Products, Journal of Engineering and Applied Sciences, 13, 6500-6504 (2018)

2. E. Khasanov, I. Gabitov, S. Mudarisov, R. Khamaletdinov, Z. Rakhimov, I. Akhmetyanov, I. Farkhutdinov, I. Masalimov, R. Musin, Justification of parameters of seed treater with an eccentrically fixed drum influencing the motion character and seed treatment modes, Bulgarian Journal of Agricultural Science, 25 (Suppl. 2), 119-128 (2019)

3. R. Sabirov, A. Valiev, L. Karimova, A. Dmitriev, D. Khaliullin, Engineering for Rural Development, 18, 211 (2019)

4. Patent USA 9,861,026 B2 (2018)

5. Patent AU 2018204535 A1 (2018)

6. Patent USA 0198622 A1 (2016)

7. Patent BY 13537 (2010)

8. Patent BY 13387 (2010)

9. Patent USA 9,675,001 B2 (2017)

10. Patent USA 2018/0271007 A1 (2018)

11. Patent USA 9,686,904 B2 (2017) 
12. Patent USA 9,992,927 B2 (2018)

13. Patent USA 0380262 A1 (2019)

14. Patent USA 9,215,840 B2 (2015)

15. Patent USA 9,357,693 B2 (2016)

16. Patent USA 10,407,256 B2 (2019)

17. Liao Yitao, Wang Lei, Liao Qingxi, Int. J. Agric. \& Biol. Eng., 10, 2 (2017)

18. Patent RU 2380876 (2010)

19. Patent RU 130777 (2013)

20. R.I. Nigmatulin, Dynamics of multiphase media. Partl (Nauka, Moscow, 1987)
21.S. Mudarisov, I. Badretdinov, Z. Rakhimov, R. Lukmanov, E. Nurullin, Computers and Electronics in Agriculture, 168 (2020)

22. R.I. Ibyatov, Theoretical Foundations of Chemical Engineering, 51, 6 (2017)

23. S. Mudarisov, E. Khasanov, Z. Rakhimov, I. Gabitov, I. Badretdinov, I. Farchutdinov, F. Gallyamov, M. Davletshin, R. Aipov, R. Jarullin, Journal of Mechanical Engineering Research and Developments, 40, 4 (2017) 